Musées, Patrimoine et Culture scientifiques et techniques

$129 \mid 2010$

mai - juin 2010

\title{
Collections universitaires et Paléontologie
}

University Collections and Palaeontology

Jérôme Thomas

URL : http://journals.openedition.org/ocim/172

DOI : 10.4000/ocim.172

ISSN : 2108-646X

Éditeur

OCIM

Édition imprimée

Date de publication : 1 mai 2010

Pagination : 28-35

ISSN : 0994-1908

Référence électronique

Jérôme Thomas, "Collections universitaires et Paléontologie », La Lettre de I'OCIM [En ligne],

129 | 2010, mis en ligne le 01 mai 2012, consulté le 19 avril 2019. URL : http://

journals.openedition.org/ocim/172 ; DOI : 10.4000/ocim.172 


\section{Collections universitaires et Paléontologie}

Jérôme Thomas *

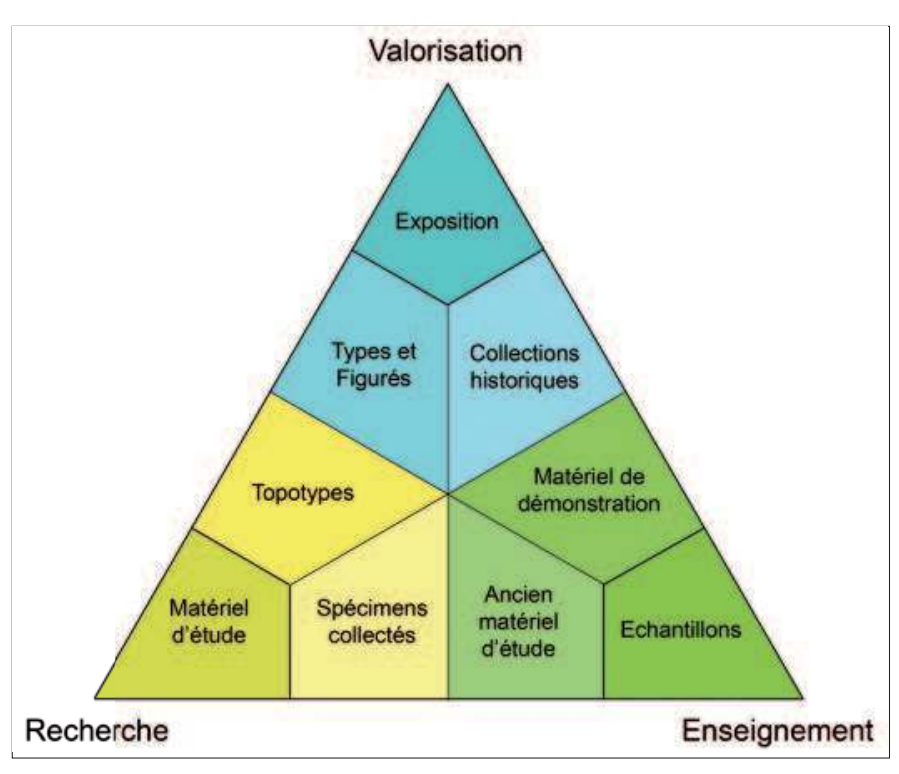

Diagramme ternaire des collections paléontologiques universitaires (modifié d'après Bailly, 2009) ๑ () Biogéosciences/Jérôme Thomas

* Jérôme Thomas est ingénieur chargé de collections muséales, UMR-CNRS 5561 Biogéosciences, université de Bourgogne jerome.thomas@u-bourgogne.fr
À partir de l'exemple du programme Trans'Tyfipal ${ }^{\circledR}$, mené par le laboratoire Biogéosciences de l'université de Bourgogne et qui a pour objectif $d$ 'inventorier tous les spécimens types paléontologiques conservés en France, l'auteur montre comment l'un des grands défis pour les musées unversitaires est de se développer en bonne harmonie entre la recherche, la formation et la valorisation.

Afin de poursuivre la réflexion engagée au colloque Universeum tenu à Toulouse en juin 2009, ce texte présente succintement un questionnement sur les collections universitaires de Paléontologie à travers l'exemple du service des collections du laboratoire de recherche Biogéosciences de l'université de Bourgogne. Dans l'ensemble de l'article, nous considérons la collection comme un regroupement thématique d'objets et non uniquement la partie patrimoniale des spécimens conservés.

Dans le cadre du programme Trans'Tyfipal ${ }^{\circledR}$, l'université de Bourgogne, et en particulier le laboratoire Biogéosciences, pilote l'inventaire des fossiles types et figurés conservés en France. La recommandation 16C $\mathrm{du}$ Code international de Nomenclature zoologique (Ride et al., $1999^{(1)}$ ), qui stipule que «les types constituant des standards de référence internationaux, les auteurs (décrivant une nowvelle espèce) devraient déposer les spécimens types dans une institution qui entretient une collection de recherche ", nous interroge premièrement sur la définition des collections liées à la recherche et dans un deuxième temps sur la propriété et la protection de ces spécimens. 


\section{Diversité des collections universitaires}

Les musées peuvent se définir autour de trois fonctions principales : préserver, étudier et communiquer (Poulot, D. (2)), le code de l'éducation en impose également trois principales aux universités françaises : l'enseignement, la recherche et la diffusion des connaissances. À partir de celles-ci, nous pouvons établir un diagramme ternaire (modifié d'après Bailly (3) dans lequel s'inscrivent des collections paléontologiques universitaires n'ayant pas toutes une vocation patrimoniale à un instant donné :

- le pôle Diffusion des connaissances (Valorisation) prend en compte les spécimens d'exposition, les collections historiques (collecteur célèbre, matériel ancien de démonstration...), les types (spécimens utilisés pour décrire une nouvelle espèce) et figurés (spécimens figurés dans une publication);

- le pôle Enseignement regroupe les échantillons collectés pour la pédagogie, le matériel de démonstration et l'ancien matériel d'étude dont l'intérêt scientifique est déclaré nul ;

- le pôle Recherche comprend les spécimens collectés par les enseignants-chercheurs et chercheurs en activité, le matériel d'étude conservé depuis de nombreuses années et les spécimens topotypes collectés avec les types et figurés (ceci afin de permettre des analyses destructives sans porter atteinte aux types). La position d'un objet pourra évoluer au cours du temps. Un spécimen d'étude peut devenir un type ou figuré après publication, un objet d'enseignement servira à l'étude historique de la pédagogie tels que les agrandissements de microfossiles d'Alcide d'Orbigny.

Différentes publications (Fara \& al. (4), Hunter \& al., (5) et Davis \& al., (6)) reconnaissent des biais entre les collections des musées, plus particulièrement dans les anciennes collections, et l'enregistrement fossile issu des fouilles. Si l'on reprend l'étude de Fara \& al., le biais apparaît dans la sous représentation du genre Vinctifer dans les collections alors qu'il est très abondant sur le site de fouille. En parallèle, les spécimens du genre Tharrhias sont aussi abondants en collection que dans les couches géologiques. Ainsi, avant cette étude, la connaissance du bassin d'Araripe au Brésil semblait sévèrement biaisée par des critères sélectifs purement esthétiques, en particulier dans les anciennes collections.

On peut donc se demander si ces anciennes collections peuvent encore être utiles pour la recherche? Rappelons que c'est entre 1909 et 1917 que Walcott fouilla les schistes de Burgess, mais qu'il faudra attendre les années 1970 pour que Whittington, Briggs et Conway-Morris réinterprètent ces spécimens conservés en collection et en fassent une révolution de la Paléontologie moderne. La réinterprétation des spécimens de la faune de Burgess, soixante années après leur mise en collection, nous montre bien que l'intérêt scientifique d'une collection peut évoluer au cours du temps, en lien avec le développement des techniques d'analyses et les concepts acquis depuis.

Ce n'est pas l'aspect esthétique ou extraordinaire (le plus gros ou le mieux préservé) qui guide l'intérêt

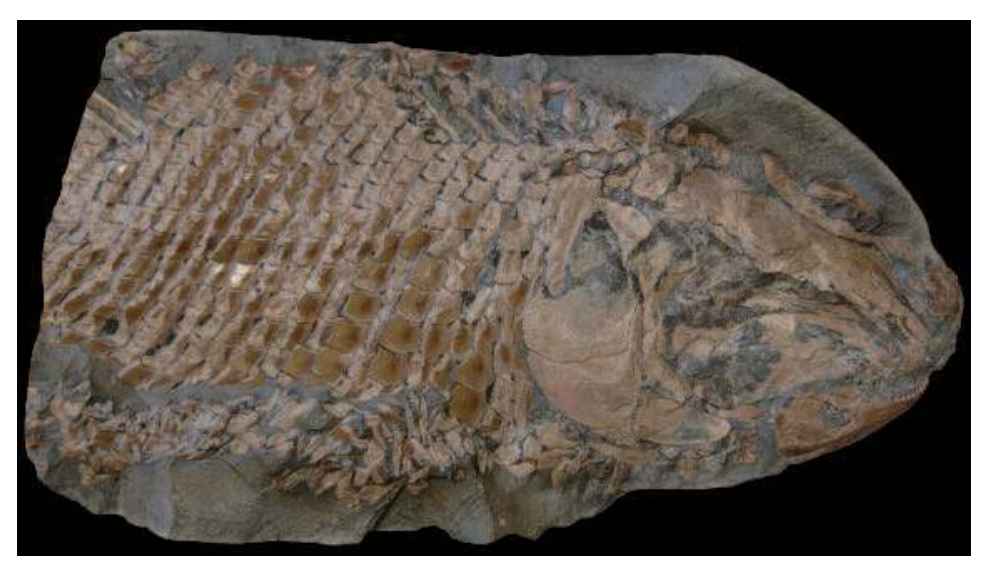

Spécimen de Lepidotes elvensis (UBGD 276166) publié par Dera G. et al., 2009. ๑) Biogéosciences/Jérôme Thomas

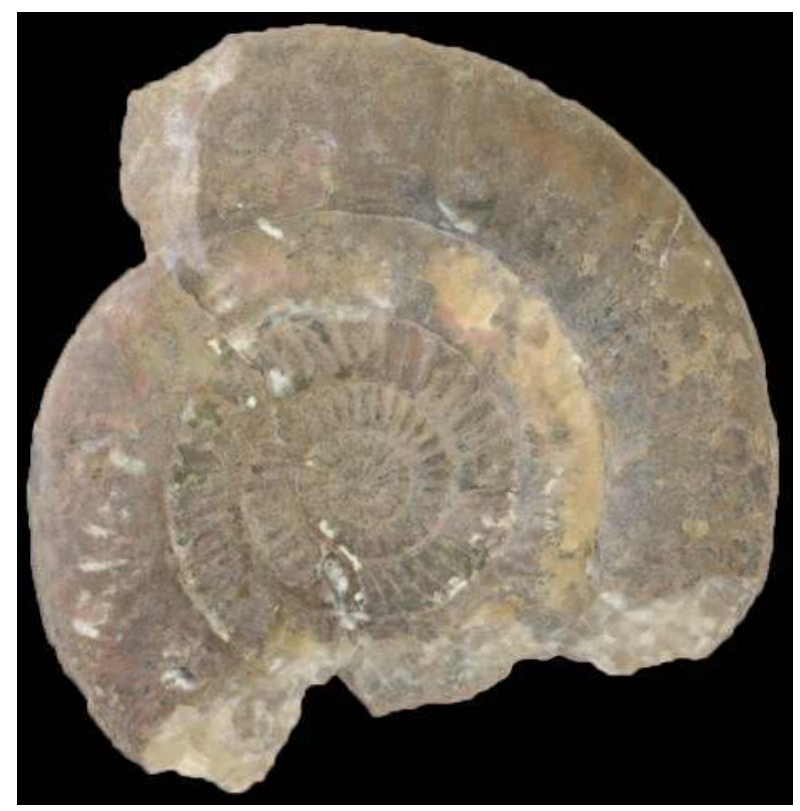

Spécimen de Plesechioceras platypleura (UBGD 276668) publié par Dommergues J.-L., 1993. ( ) Biogéosciences/Morgane Brosse 

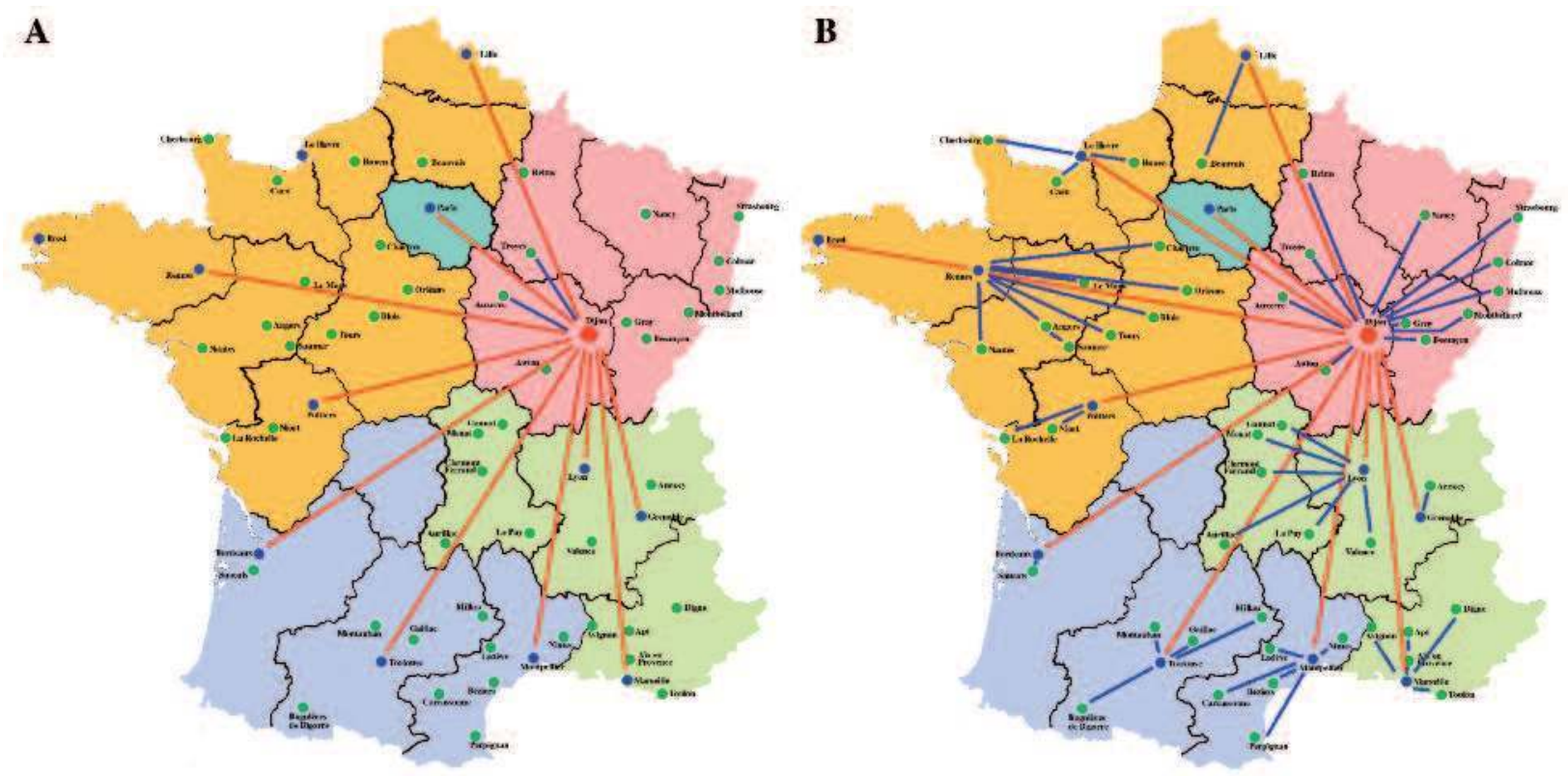

A : État des lieux du maillage du programme Trans'Tyfipal en mars 2010 $B$ : Projet de maillage final (de nombreux musées ne figurent pas encore) ( Trans'Tyfipal/Jérôme Thomas

scientifique. Prenons l'exemple de deux spécimens conservés à l'université de Bourgogne. Pour le poisson fossile UBGD 276166, son importance réside dans le micro-prélèvement effectué sur l'une de ses dents afin d'étudier l'évolution des paléotempératures marines au Jurassique (Dera, G. et al. (7)). En ce qui concerne l'ammonite UBGD 276668, il s'agit d'un spécimen type ayant servi à décrire la nouvelle espèce Plesechioceras platypleura Dommergues J.-L. 1993 (Dommergues, J.-L (8)). Pourtant, aux yeux du profane, cette pièce incomplète parait banale.

Nos réserves sont remplies de spécimens anciens dont nous ne pouvons pas clairement définir l'intérêt pour l'avenir. La politique actuelle concernant la Paléontologie se découpe en deux axes principaux : biologique par la compréhension des mécanismes de l'évolution, de la systématique, de la biodiversité et géologique par la biostratigraphie, la taphonomie et la géochimie. Ces orientations scientifiques nécessitent cependant des informations précises, en particulier en ce qui concerne la position stratigraphique du spécimen (origine du fossile dans la coupe géologique documentée et donc son âge supposé).

Ces constats portant sur l'évolution de l'intérêt scientifique nous permettent de lancer un débat ouvert sur les collections universitaires : doit-on tout conserver et quels critères de tri pouvons-nous établir ? Peut-on jeter le matériel ancien en raison du manque de précision stratigraphique ou de l'absence de documentation ?
Doit-on tout protéger au titre du patrimoine en oubliant la fonction première scientifique de certains spécimens pouvant conduire à la destruction de ces pièces pour l'obtention de données ?

Cette notion de sélection s'applique également pour l'acquisition de spécimens. Que ce soit par la collecte des chercheurs ou via le don de collections par des particuliers (dans le contexte actuel oublions l'achat car rares sont les universités disposant de fonds adaptés), la politique d'acquisition de spécimens du laboratoire Biogéosciences spécifie des critères comme la précision des données (plus particulièrement concernant la stratigraphie) et l'adéquation entre le matériel et les projets de recherche. Ainsi, récemment, nous avons accepté une collection de plus de 5000 pièces et refusé une autre par manque de précision dans les informations de géolocalisation (nous avons cependant suggéré au propriétaire de cette superbe collection régionale de s'adresser à nos collègues des différents muséums de la région Bourgogne).

Cette politique n'est praticable que dans des universités possédant encore des laboratoires en Paléontologie. Pour celles dépourvues de structures de recherche adaptées, une approche beaucoup plus muséale est envisageable. Cependant, il ne faut pas oublier le passé de l'institution où un ancien laboratoire a pu « entasser » du matériel d'étude difficilement exploitable pour la médiation de la recherche 
et de la science. Ces collections sont donc à inscrire dans un réseau de diffusion, afin que ces spécimens puissent servir à des chercheurs extérieurs à la structure de dépôt. Trans'Tyfipal ${ }^{\circledR}$ s'est développé pour répondre à cette attente concernant les types et figurés. Rappelons que ce programme s'est restructuré autour des principaux établissements conservant ces spécimens référentiels. Il forme ainsi un maillage national, qu'une seconde phase de déploiement complètera par un maillage secondaire régional afin de mettre en réseau l'intégralité des établissements possédant des types et figurés fossiles. L'actualisation du catalogue des collections paléontologiques françaises (Prieur et al. (9)) débute afin de mieux appréhender l'ampleur de ces collections en France.

En ce qui concerne les spécimens patrimoniaux, peut-être qu'une plate-forme web présentant les spécimens empruntables pour exposition et intégrant les collections des universités et des musées serait à créer ?

Cette notion de réseau nous paraît essentielle car elle favorise des rapprochements entre établissements à travers le partage des expériences et des compétences.

\section{Propriété et protection}

La propriété d'un objet est relativement simple à définir dans le cadre d'un achat ou d'un don, ainsi que pour un établissement dirigé par une seule tutelle.

Cependant, les principaux laboratoires de Paléontologie ne répondent pas à ces critères. Soit le matériel est collecté, soit ces structures de recherche sont pilotées par au moins deux tutelles dont la priorité ne va pas forcément à ces questions de conservation. Rappelons qu'avant même l'arrivée au laboratoire, la pièce récoltée appartient tout d'abord au propriétaire du sol sur lequel a été prélevé le spécimen, qui peut en faire don à un organisme public. Par la suite, le scientifique auteur de la description de la pièce développe une forme de propriété intellectuelle, mais il ne détient nullement la propriété de l'objet. Celui-ci appartient aux tutelles de l'unité de recherche dans laquelle il travaille (Rainette, C. $\left.{ }^{(10)}\right)$. Par exemple, pour l'Unité Mixte de Recherche (UMR) Biogéosciences, les objets collectés actuellement devraient appartenir aux établissements publics à caractère scientifique et technologique qui forment les tutelles de ce laboratoire, donc en toute logique au Centre National de la Recherche Scientifique (CNRS) et à l'université de Bourgogne.
Ainsi, si nous voulions être très procéduriés, certaines collections déposées par des chercheurs dans des musées devraient revenir dans leurs universités d'origine. Cependant, ces dépôts «sauvages » ont permis de sauvegarder des collections de grande valeur scientifique, là où des structures de recherche les auraient abandonnées. Ce qui démontre bien que les procédures administratives doivent aussi s'adapter au pragmatisme des situations.

Si l'on se penche sur la question de la protection des collections paléontologiques universitaires, nous pouvons reprendre le diagramme ternaire en établissant une hiérarchie dans les degrés de protection.

En ce qui concerne les collections de valorisation, il n'y a aucune ambiguïté à avoir, il faut absolument appliquer la meilleure protection possible. Ces collections sont à vocation patrimoniale en application de l'article Ll du Code du Patrimoine par leur intérêt historique, scientifique ou technique. Cette reconnaissance patrimoniale permettrait d'inscrire ce matériel collecté (ou plus rarement acheté) à l'inventaire du domaine public mobilier de la personne publique propriétaire des spécimens, rendant ces derniers inaliénables, insaisissables et imprescriptibles (code général de la propriété des personnes publiques). L'application de la loi Musée de France est également envisageable, mais de nombreuses universités n'ont pas d'espace d'exposition permettant d'assurer l'accueil du public et, de fait, ne peuvent raisonnablement pas demander cette appellation.

Les spécimens dits de recherche peuvent être détruits pour des besoins scientifiques ou patrimonialisés après publication ou pour des raisons historiques (spécimens collectés par un chercheur célèbre). La constitution de ces collections a (ou a eu) un coût non négligeable se chiffrant à des milliards d'euros en ne comptant que les frais pour les missions de collecte. Ces collections commencent à être considérées comme de véritables outils scientifiques au même titre qu'un gros équipement. Cependant, elles ne disposent d'aucun moyen de protection. L'inscription à l'inventaire, qu'il soit sous le régime musée de France ou sous la domanialité publique, risque de s'avérer très contraignante si l'on doit attendre l'accord des commissions de déclassement et déontologiquement parlant peu raisonnable si l'on doit protéger pour mieux détruire par la suite. Une solution envisageable serait une forme de protection avec un déclassement par une commission interne composée de conservateurs et scientifiques de l'établissement, plus facilement consultable qu'une commission d'acquisition et de restauration-conservation. 
De nombreux travaux sont en cours un peu partout, partageant les mêmes questions : quels sens pouvons-nous donner à nos collections ? Quels liens pouvons-nous établir entre ces collections et les laboratoires de recherches ? Quel est l'objectif général d'un musée au sein d'une université ? Quelles formes peut-il prendre ?... L'université de Bourgogne a des particularités intéressantes pour éclairer ces questions par les activités quelle propose et les structures qu'elle a à sa disposition :

- les collections sont un des éléments de culture scientifique de l'université ;

- il existe plusieurs cursus en liaison avec les collections : Master professionnel de Muséologie et de Médiation culturelle, programme court de vulgarisation des sciences et de restauration du patrimoine ; - un laboratoire en Sciences de l'Information et de la Communication conduisant des recherches et proposant des séminaires sur le sujet - plusieurs doctorats et programme de recherche sont en cours ; - des partenariats avec les structures régionales.

Des collections.... au sein de l'université de Bourgogne. Ce terme n'est pas très habituel dans les couloirs d'une université car on pense alors à quantité d'objets obsolètes vis-à-vis de la science actuelle et des recherches contemporaines.

La faculté de Droit a été créée à Dijon en 1722. En 1808, la faculté des Sciences, de Médecine et de Pharmacie a été mise en place ainsi que la faculté des Lettres. C'est en 1957 que la plupart des activités universitaires rejoignent la colline Montmuzard. L'enseignement et la recherche scientifique nécessitaient et nécessitent encore un matériel spécialisé de démonstration, d’observation et d'expérimentation, certains domaines plus que d'autres, notamment en Biologie végétale et en Géologie. Ce matériel acquis depuis 1808 n'a pas été entièrement conservé, mais certains éléments ont été sauvés. Ces collections ont donc un intérêt patrimonial certain en illustrant l'histoire des sciences mais aussi l'histoire de l'enseignement.

Depuis plusieurs années, l'université a pris conscience de limportance de ce patrimoine et, peu à peu, un réseau s'est construit afin de faire connaître, de conserver et de mettre en valeur ces objets très différents dans de nombreuses composantes. Le patrimoine peut aussi se décliner au présent. En effet, les objets, les machines utilisées pour la recherche évoluent très vite. Si tout est jeté, alors la seule trace des recherches sera la publication et toute une partie de l'information sera perdue. En Bourgogne, depuis 2007, l'université est chargée de mettre en place un inventaire du patrimoine scientifique et technique contemporain en sensibilisant les établissements d'enseignement supérieur et de recherche mais aussi les entreprises à la sauvegarde dans le cadre du réseau PATSTEC (Patrimoine scientifique et technique contemporain) initié par le ministère de la Recherche via le musée des Arts et Métiers.

De nombreuses actions ont été mise en place depuis plusieurs années grâce à des personnels passionnés au sein des différentes composantes. Ce réseau a été valorisé par la mise en place de la Mission à la culture scientifique en 2002. Dès lors, des expositions ont permis de mettre en valeur le patrimoine de l'université comme par exemple : Objets raisons, objets passions, première exposition destinée à sensibiliser autant en interne qu'en externe à la problématique du patrimoine et qui, après être restée au centre culturel de l'université, a itinéré dans plusieurs musées (muséum d'Histoire naturelle de Dijon, musée Buffon à Montbard).

Depuis 2006, le ministère de la Culture a mis à disposition un conservateur d'État au sein de l'université avec pour but d'augmenter la coordination entre les différents acteurs et d'assurer au
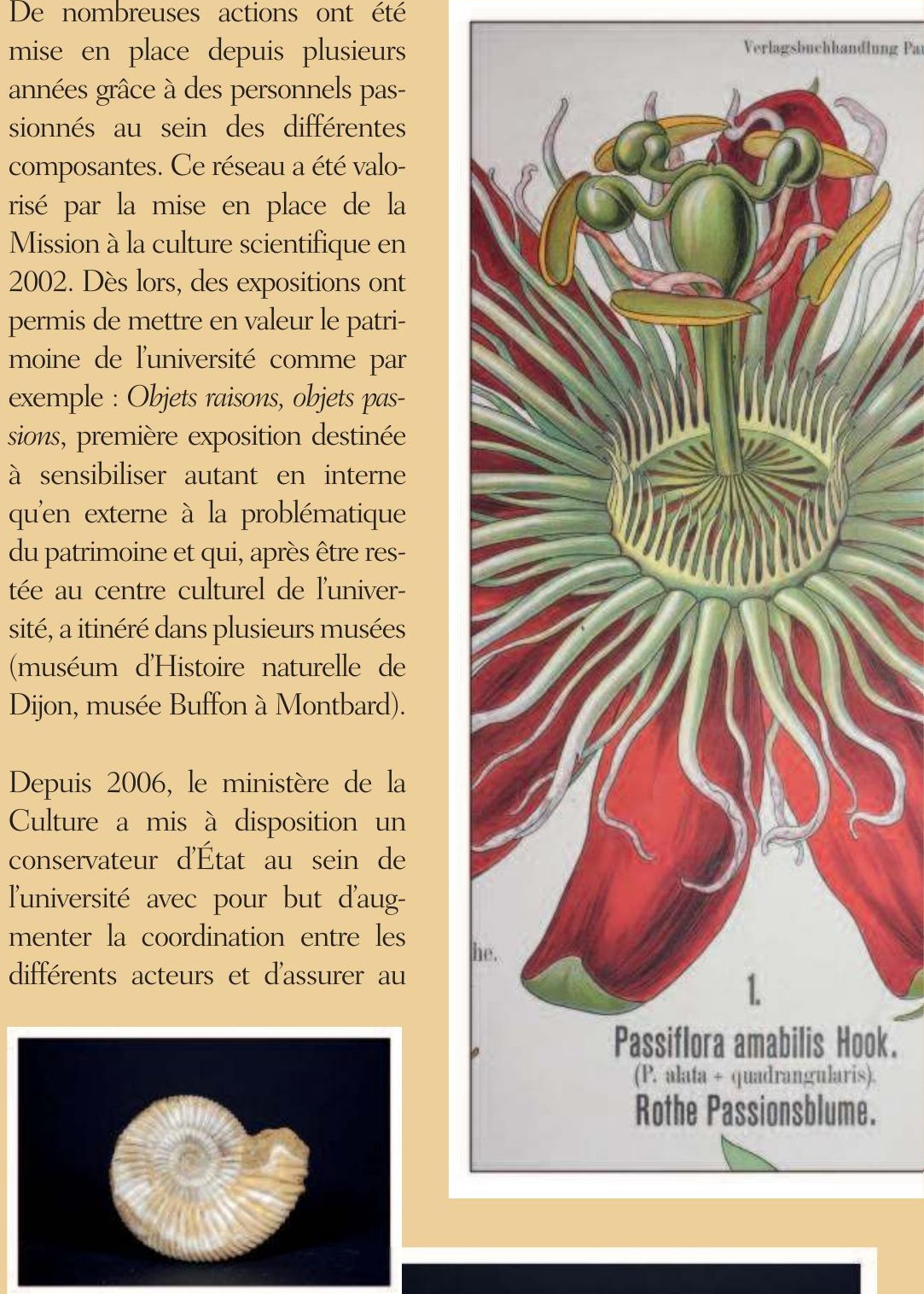

Passiflora amabilis Hook. (P. alata + quadrangularis). Rothe Passionsblume.

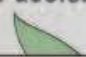

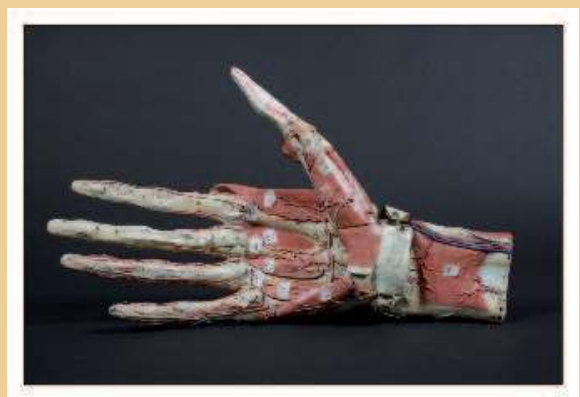

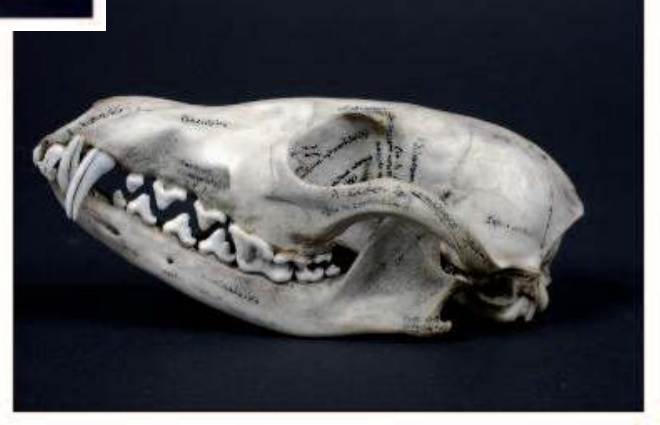

๑) pour l'ensemble des photographies/Vincent Arbele 


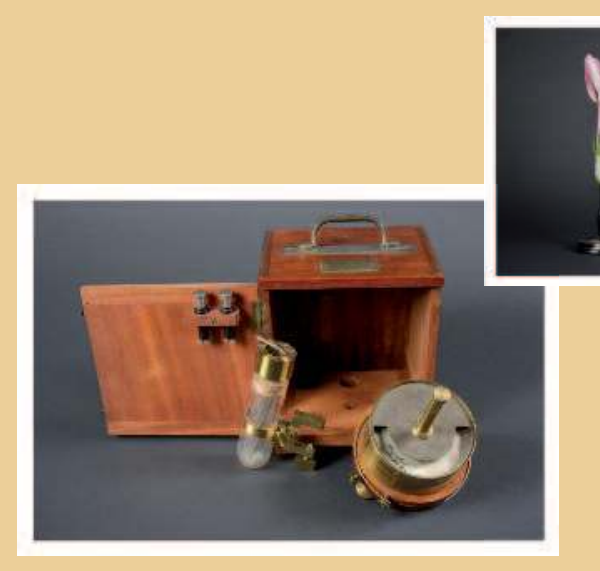

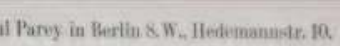
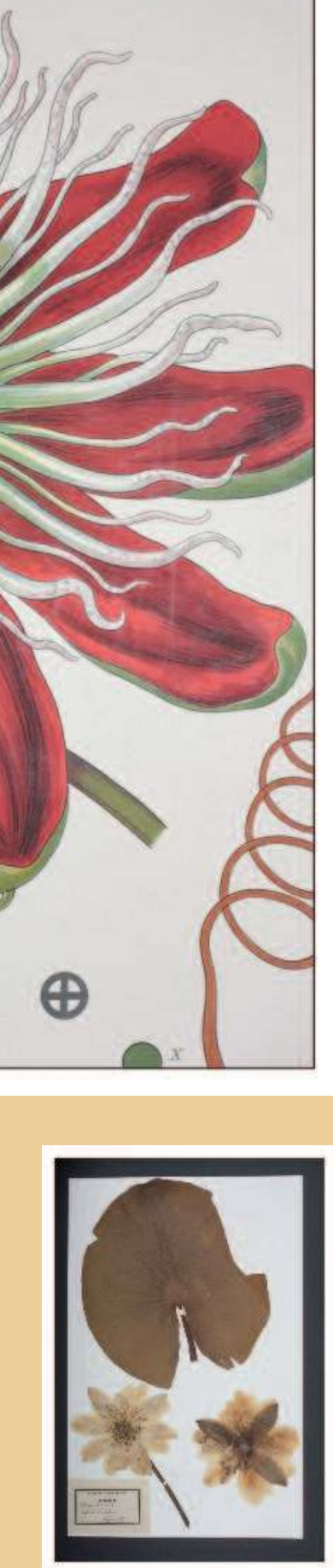

mieux la conservation de ce patrimoine. Afin de faire connaître « les trésors » de l'université, plusieurs expositions ont été mises en place au sein du campus mais aussi dans les musées de la ville de Dijon : Autopsie d'un livre ancien; Objectifs pôles; Hautes tensions; Du réel au virtuel : où sont passées les collections?

Il s'agit, à chaque fois, de mettre les collections en relation avec la recherche ou les enseignements de l'université. Les collections sont des supports à des actions de recherches actuelles pour la plupart et ne sont pas seulement et toujours des objets « morts ». Elles servent encore à l'enseignement en Botanique ou en Médecine par exemple. Il est important de souligner ce lien et de le mettre en valeur. Bien entendu, les collections sont la trace d'une histoire mais ce n'est pas n'importe quelle histoire, c'est celle de l'université de Bourgogne avec toutes ses spécificités. Ce n'est qu'en s'appuyant sur ce postulat que nous pourrons faire comprendre aux différents personnels quil est important de garder certaines des pièces quils côtoient. D'autres types d'actions ont été mises en place comme par exemple l'édition de fascicules d'histoire des sciences ou encore des stages « Faire l'histoire de sa science » à destination des doctorants. Par ailleurs, un catalogue, Les collections scientifiques de l'université de Bourgogne, récemment édité, présente les objets rassemblés par disciplines : Médecine (plus de 500 pièces anatomiques en cire, plâtre, carton-pâte, plastique et même en os, véritables outils pédagogiques destinés parfois encore aujourd'hui à l'enseignement de l'Anatomie), Physique (près de 400 instruments d'expérimentation, dont certains en cuivre et bois datent du XVIIIe siècle), Paléontologie (une collection de 950000 spécimens de Paléontologie et 50000 de Géologie constituée dès le début du XIXe siècle), Botanique (404 échantillons des algues marines, 150 modèles de fleurs, 300 planches d'herbier, banques de données et

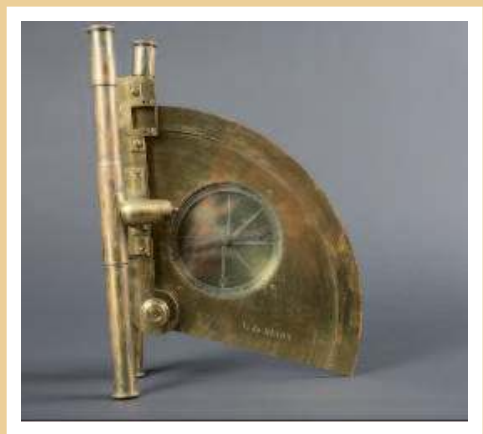

outils précieux de formation et d'aide à la détermination), Zoologie (planches anatomiques anciennes, squelettes, coquillages, en tout plus de 2000 spécimens, témoins de la biodiversité et supports d'enseignement et d'études scientifiques), Documentaion (8 000 ouvrages, dont 5000 datant d'avant 1811, provenant de toutes les disciplines).

Les collections de l'université n'ont pas pour but de devenir des objets d'un nouveau musée des sciences. La Bourgogne possède 3 muséums qui jouent leur rôle de diffusion du savoir et qui irriguent le territoire ; l'université de Bourgogne est partenaire de ces 3 établissements. Le lien entre nos collections et la recherche apporte une dimension particulière qui doit être mise en avant et qui leur donne du sens. L'université de Bourgogne n'aura probablement jamais de musée dans ses locaux, même si un espace consacré aux sciences non naturelles est absent du paysage régional. Il nous faut pourtant trouver des solutions pour ne pas perdre l'histoire de la recherche et pour garder des éléments qui serviront à la recherche de demain.

Le musée virtuel par le biais de site Internet est une des solutions déjà utilisée par le réseau PATSTEC notamment. De son côté, l'université de Bourgogne a développé 2 types de films : un premier format de 2 minutes très facile à voir sur un site qui fait découvrir un univers à part, celui des collections par la rencontre entre un candide (universitaire, personnel administratif...) et une personne chargée des collections, et un second de 5 à 6 minutes qui présente plus particulièrement une collection (celui sur la Paléontologie a débuté la série, celui sur les livres anciens va sortir et d'autres sont en préparation notamment en Botanique).

Un patrimoine toujours vivant et toujours à découvrir...

DANiEl Raichvarg

Vice-président Cultures et Cités, université de Bourgogne

Marie-Laure Baudement

Conservateur du Patrimoine, université de Bourgogne 
Exceptés certains modèles de démonstration qui peuvent être patrimonialisés, les spécimens d'enseignement n'ont pas à être protégés vu le grand nombre de manipulations par les étudiants et les enseignants. De plus, de nombreuses pièces finissent cassées ou abîmées, si ce n'est pas volées comme trophée (certains crânes édentés en sont malheureusement d'excellents témoins). À ce titre, aucune protection particulière ne semble nécessaire.

Du point de vue quantitatif, nous sommes bien loin du constat de patrimonialisation abusive : «Toujours plus. Plus de pièces, plus de personnel, plus d'argent. La notion même de patrimoine est devenue exponentielle»(Gervereau, L. (11)). En effet, sur 1 million de spécimens environ, on estime à 9000 le nombre d'objets dont 8500 types et figurés à patrimonialiser, la collection pédagogique regroupe environ 2000 pièces, le reste (ce qui fait tout de même 989500 spécimens) compose le matériel destiné à la recherche, donc non patrimonial.

Concrètement et en réponse aux objectifs de protection énoncés, ces différentes catégories de collections influent sur la gestion des spécimens fossiles du laboratoire Biogéosciences et imposent des critères d'accessibilité diversifiés en attendant une protection officielle. Ainsi, nous comptons cinq principaux espaces de conservation. La typothèque, seulement accessible au conservateur, accueille les types et figurés. Le Jardin Jurassique reçoit les autres spécimens de valorisation formant ainsi une salle d'exposition, accessible à tout public, présentant la Paléontologie et la Géologie régionale, des spécimens relatant l'historique du laboratoire et des moulages comme ceux liés à l'évolution humaine. Le matériel d'étude actif, c'est-à-dire en cours d'utilisation pour des recherches actuelles, est conservé dans une salle mise à disposition des chercheurs où chacun d'entre eux a un espace délimité par le conservateur. Le matériel d'étude passif est conservé dans une salle accessible aux chercheurs sur demande auprès du conservateur. Enfin, le matériel pédagogique est géré par les enseignants dans une salle de travaux pratiques.

\section{Conclusion}

Les collections paléontologiques universitaires se composent de spécimens anciens, souvent en très grand nombre, et de lots en cours de constitution fortement liés à la recherche. Étant généralement conservés dans des espaces finis (certains ayant même tendance à se réduire), avec des moyens humains limités, il nous faudra répondre rapidement à deux questions majeures : que faire de l'ancien et comment intégrer le matériel entrant qui composera pour une certaine partie les collections patrimoniales de demain ? Notons tout de même que suite à l'apparition des différents postes «Information scientifique et technique, collections patrimoniales » de la BAP F, différents emplois ont été créés ou réorientés selon ces profils de professionels de la conservation au sein de certains laboratoires.

Actuellement, même si les musées d'Histoire naturelle sont de bons modèles pour la conservation des collections, leur objectif se concentre plus particulièrement vers l'exposition et non le développement de nouvelles collections de recherche scientifique. Les laboratoires et leurs tutelles doivent donc mettre en place leurs collections en interaction avec les chercheurs. L'un des plus grands défis pour les structures de conservation universitaires est de se développer en bonne harmonie entre la recherche, l'enseignement et la valorisation de la recherche, ceci en même temps que les universités acquièrent leur autonomie administrative et financière et que les musées et centres de culture scientifique et technique (CST) poursuivent leurs activités de médiation scientifique. Un tel développement doit passer par une reconnaissance des tutelles aussi bien par des apports financiers qu'humains, et également par la mise en place de critères de promotion du personnel dédié. Ces actions universitaires ne sont pas à opposer à celles des musées et centres de CST, au contraire, elles doivent être complémentaires, voire mutualisables.

\section{Notes}

(1) Ride, W.-D.-L., Cogger, H.-G., Dupuis, C., Kraus, O., Minelli, A., Thompson, F.-C. et Tubbs, P.-K. International code of zoological nomenclature, fourth edition. London : The International Trust for Zoological Nomenclature, 1999, $306 \mathrm{p}$

(2) Poulot, D., 2005. Musée et muséologie. Paris : La Découverte, 2005, $122 \mathrm{p}$.

(3) Bailly M. Gestion et valorisation des « collections » scientifiques universitaires. Mémoire du Master 2 Géobiosphère, sous la direction de MarieLaure Baudement et Jérôme Thomas, université de Bourgogne, 2009, $53 \mathrm{p}$.

(4) Fara, E., Saraiva, A., Campos, D.-A., Moreira, J.-K.-R., Siebra, D.-C. et Kellner, A.-W.-A. Controlled excavations in the Romualdo Member of the Santana Formation (Araripe Basin, Lower Cretaceous, northeastern Brazil) : stratigraphic, palaeoenvironmental and palaeoecological implications. Palaeogeography, Palaeoclimatology, Palaeoecology, 218 (1-2), 2005, pp. 145-160,

(5) Hunter, A.-W. et Donovan, S.-K. Field sampling bias, museum collections and completeness of the fossil record. Lethaia, vol. 38, 2005, pp. 305-314. 
(6) Davis, E.-B. et Pyenson, N.-D. Diversity biases in terrestrial mammalian assemblages and quantifying the differences between museum collections and published accounts : A case study from the Miocene of Nevada. Palaeogeography, Palaeoclimatology, Palaeoecology, 250, 2007, pp. 139-149.

(7) Dera, G. et al. Water mass exchange and variations in seawater temperature in the NW Tethys during the Early Jurassic : Evidence from neodymium and oxygen isotopes of fish teeth and belemnites. Earth and Planetary Science Letters, 286, 2009, pp. 198-207.

(8) Dommergues, J.-L. Les ammonites du Sinémurien supérieur de Bourgogne (France) : biostratigraphie et remarques paléontologiques, Revue de Paléobiologie, 12 (1), 1993, pp. 67-173.
(9) Prieur, A. (avec la collaboration de Gaillard, M. et Vigne, H.) Catalogue des collections paléontologiques françaises. Office national de gestion des collections paléontologiques françaises, université Claude Bernard Lyon 1, 1980, 307 p.

(10) Rainette, C. (en collaboration et sous la direction scientifique de Cornu, M. et Wallaert, C.) Guide juridique sur le patrimoine scientifique et technique. Paris : L'Harmattan, 2008, 186 p.

(11) Gervereau, L. Vous avez dit musées? Tout savoir sur la crise culturelle. Paris : CNRS Éditions, 2006. 